\title{
Eine Beziehung entwickelt sich - Lichtbilder und neue Produktionsweisen
}

Ein Besucher, der sich 1896 für Maschinen und industrielle Produktion interessierte, lenkte auf dem Gelände der Allrussischen Industrie- und Kunstausstellung (Vserossijskaja promyšlennaja $i$ chudožestvennaja vystavka) in Nižnij Novgorod seinen Schritt wohl als erstes zu den Pavillons der Abteilungen neun und zwölf: zu den „Betriebs-, Fabrik- und Gewerbeerzeugnissen“ und „Maschinen, Apparaten, Maschinenbau und Elektrotechnik". ${ }^{1}$ In den weitläufigen, lichtdurchfluteten Hallen der eigens angefertigten Ausstellungsgebäude aus Glas und Stahl ${ }^{2}$ bewunderte er eine Vielzahl an Ständen, an denen Unternehmen ihre Produktion eindrücklich präsentierten und dafür enorme Kosten auf sich nahmen. ${ }^{3}$ Wie der Name bereits zeigt, waren die Exponate der zwölften Abteilung in erster Linie große Dampfmaschinen, Generatoren, Pflüge oder Dreschmaschinen. ${ }^{4}$ Die Ausstellungsstände der neunten Abteilung zeigten riesige, aus Fässchen und Flaschen zusammengesetzte Säulen in den Farben der Flagge des Zarenreichs, ${ }^{5}$ andere Unternehmen stellten Lattenroste, Kinderwägen oder Stoffe aus ${ }^{6}$ und die Russische-Amerikanische

1 Ein Ziel der Ausstellungen war es, neben Ausländern auch russische Besucher für die Wirtschaft des Zarenreichs zu begeistern und ihr Vertrauen in die heimische Industrie zu stärken. Joseph Bradley: Voluntary Associations, Civil Culture and Obshchestvennost' in Moscow, in: Edith Clowes; Samuel Kassow; James West (Hrsg.): Between Tsar and People. Educated Society and the Quest for Public Identity in Late Imperial Russia, Princeton 1991, S. 131-148, S. 146.

2 o. A.: Vserossijskaja promyšlennaja i chudožestvennaja vystavka v Nižnem-Novgorode 1896 goda, Odessa 1896, S. 16, 35-36.

3 A. A. Efron: Vsemirnaja vystavka i russkie èksponenty. L'exposition universelle et les exposants russes. S" ukazatelem Fabrik i zavodov, Sankt-Peterburg 1886, S. 112-113.

4 RGB: Izo 29184-54; MK XII-529: o. A.: Vidy vserossijskoj chudožestvennoj promyšlennoj vystavki 1896 g. v Nižnem Novgorode, Moskva (vermutl.) 1896, S. 178-180. Zur Bedeutung von Maschinen auf Industrieausstellungen siehe auch: Thomas Großbölting: Im Reich der Arbeit. Die Repräsentation gesellschaftlicher Ordnung in den deutschen Industrie- und Gewerbeausstellungen 1790-1914, München 2008, S. 342-348.

5 Die Verknüpfung von industrieller Entwicklung, Nationalismus und Patriotismus im Rahmen von Ausstellungen war im Zarenreich während des 19. Jahrhunderts sehr populär. Joseph Bradley: Pictures at an Exhibition. Science, Patriotism, and Civil Society in Imperial Russia, in: Slavic Review, Jg. 67/2008, Heft 4, S. 934-966, S. 954.

6 Vidy vserossijskoj chudožestvennoj promyšlennoj vystavki, S. 139-140, 188, 191, 193. 
Gummiwarenfabrik aus St. Petersburg faszinierte die Besucher mit einem riesigen aufgeschichteten Berg aus Gummischuhen. ${ }^{7}$ Häufig beeindruckten die Hersteller mit der schieren Masse oder Größe ihrer Exponate - dieser Gigantismus war ein verbreitetes Phänomen bei russischen Ausstellern. ${ }^{8}$ Eine andere Möglichkeit war, möglichst ausgefallene Ausstellungsstücke zu präsentieren, so zeigte die Parfüm und Seifenfabrik Brokar i Ko auf der Allrussischen Industrie- und Kunstausstellung in Moskau im Jahr 1882 einen Brunnen, aus dem Kölnisch Wasser floss. ${ }^{9}$

$\mathrm{Zu}$ den meisten Ständen gehörten neben ungewöhnlichen Exponaten aus der jeweiligen Produktpalette auch visuelle Darstellungen. Am verbreitetsten waren Gesamtansichten der jeweiligen Fabrik, wahlweise als Zeichnung oder als Fotografie..$^{10}$ Neben Panoramen zeigten Unternehmen auch ganze Fotoalben an ihren Ständen. ${ }^{11}$ Der Nachteil war, Alben waren verhältnismäßig teuer in der Herstellung, damit wertvoll, und sollten darum nicht unbeaufsichtigt ausliegen. Unbedenklicher war es, die Aufnahmen zu rahmen, sie neben vom Unternehmen errungenen Preisen aufzuhängen oder wie die Kanonenfabrik aus Perm (Permskij pušečnyj zavod) spezielle Fotovitrinen mit beweglichen Rahmen zu nutzen, die die Besucher wie ein Buch durchblättern konnten. ${ }^{12}$ Fotografische Aufnahmen ermöglichten es den Betrieben neben ihren fertigen Produkten dem Besuchern gleichzeitig einen Eindruck von den

$7 \quad$ M. A. Orlov: Vsemirnaja Parižskaja vystavka 1900 goda v illjustracijach i opisanijach. Illjustrirovannoe priloženie k „vestniku inostrannoj literatury“ 1900 g., Sankt-Peterburg 1900, S. 164 .

8 Auch wenn die Unternehmer ihre Firmen auf Weltausstellungen präsentierten, griffen sie gerne zu diesem Stilmittel. Jurij Anatol'evič Nikitin: Vystavočnaja Architektura Rossii XIX-načala XX. v., Sankt-Peterburg 2014, S. 161.

9 Ėleonora Glinternik: Reklama v Rossii XVIII-pervoj poloviny XX veka. Opyt illjustrirovannych očerkov, Sankt-Peterburg 2007, S. 79 .

10 Beispielsweise: Vidy vserossijskoj chudožestvennoj promyšlennoj vystavki, S. 145. Auch deutsche und französische Unternehmer zeigten diese Ansichten besonders gerne an ihren Ausstellungsständen. Zu Deutschland: Bodo von Dewitz: „Die Bilder sind nicht teuer und ich werde Quantitäten davon machen lassen!“ Zur Entstehungsgeschichte der Graphischen Anstalt, in: Klaus Tenfelde (Hrsg.): Bilder von Krupp. Fotografie und Geschichte im Industriezeitalter, München 1994, S. 40-66, S. 41; zu Frankreich: Céline Assegond: Naissance de la "photographie industrielle“. Regards sur l'industrie du chocolat à Paris et ses environs, Vortrag in: „Lindustrie, patrimoine et culture“ Séminaire de recherche, Région Île-de-France, Université Paris I Panthéon-Sorbonne, 24.02.2012, URL: http://epi.univ-parisı.fr/8078440o/o/fiche_pagelibre/\&RH=epi-o3oMMooo2v38\&RF=epi-03o-MMooo2v310 (zuletzt eingesehen am 23.04.14).

11 CGA Moskvy f. 318, op. 1, d. 971, 1. 98. Es handelt sich bei diesem speziellen Zitat zwar um Alben für die Weltausstellung in Turin 1911, das Beispiel zeigt jedoch, dass Fotoalben prinzipiell ein übliches Exponat waren, um Unternehmen zu präsentieren.

Vidy vserossijskoj chudožestvennoj promyšlennoj vystavki, S. 158. 
Produktionsbedingungen und -kontexten zu vermitteln. Besonders Innenund Außenansichten der Fabriken waren beliebt. ${ }^{13}$ Auch mit gedruckten Fotografien illustrierte Broschüren gehörten zu den Materialien, mit denen russische Betriebe sich bei einem interessierten Publikum profilierten. ${ }^{14}$

Bereits die erste Weltausstellung $185^{1}$ in London stellte eine Bühne für die neue Abbildungstechnik dar, ${ }^{15}$ und in den folgenden Jahren etablierten sich fotografische Abzüge als ein fester Bestandteil unternehmerischer Selbstdarstellung. Dies war nur eine unter vielen Funktionen, die die neuen Bilder in Unternehmen übernahmen. Im Folgenden wird dargestellt, wie sich die Beziehung zwischen dem neuen Medium der Fotografie und Unternehmern entwickelte und welche Praktiken sich durch die neue Technik veränderten. Ihre dokumentarische Qualität machte die Fotografien besonders interessant, um sie in den internen Abläufen eines Unternehmens einzusetzen. ${ }^{16}$ Ein mindestens ebenso wichtiger Antrieb für die Verwendung der neuen Bilder war das Interesse der Industriellen, ihre Firma in der Öffentlichkeit zu präsentieren, sei dies auf der Bühne der Weltausstellungen oder gegenüber lokalen Kooperations- und Geschäftspartnern. ${ }^{17}$

\subsection{Fotografen und Unternehmen}

In welchem Jahr ein Fotograf die erste Aufnahme machte, die heute als Industriefotografie bezeichnet werden könnte, ist unklar. ${ }^{18}$ Auffallend ist

13 Vidy vserossijskoj chudožestvennoj promyšlennoj vystavki, S. 173.

14 MKMZ: o. A.: The Saint-Louis International Exhibition. The Kolomna Machine Works Company, Kolomna 1904. Die gleiche Publikation erschien sowohl auf Englisch als auch auf Russisch.

15 Sarah Bassnett: Exhibitions of Photography, in: John Hannavy (Hrsg.): Encyclopedia of Nineteenth-Century Photography, Bd. 1, New York 2008, S. 508-510, S. 508.

16 Kathrin Kohle: Industrie und Technik, in: Ludger Derenthal; Christine Kühn (Hrsg.): Ein neuer Blick. Architekturfotografie aus den Staatlichen Museen zu Berlin, Berlin 2010, S. $53-56$, S. 53 .

17 Ulrich Pohlmann: Industriebilder, in: ders.; Johann Georg Prinz von Hohenzollern (Hrsg): Eine neue Kunst? Eine andere Natur! Fotografie und Malerei im 19. Jahrhundert, München 2004, S. 236-237, S. 237.

18 Teilweise wird in der Forschungsliteratur ein Portrait Alfred Krupps (1812-1887) von 1849 genannt. Auf der Weltausstellung in London 1851 präsentierte ein Katalog die Fotografie eines Dampfhammers von Nasmyth von Claude-Marie Ferrier (1811-1889). Andere Autoren nennen die Fotografien von Charles Blacker Vignoles (1793-1875) und Roger Fenton (1819-1869), die $185^{2}$ den Brückenbau über den Dnjepr bei Kiew fotografisch festhielten. Gerade Eisenbahnen stellten in der Frühzeit der Industriefotografie ein sehr beliebtes Motiv dar. Howard Bossen: Eine gemeinsame Geschichte erschaffen. Zur 
jedoch, dass sowohl in Großbritannien, Frankreich, Deutschland, den Niederlanden als auch im Zarenreich erst ab den 186oer Jahren vermehrt fotografische Aufnahmen im Kontext von Fabriken zu finden sind. ${ }^{19}$ Die ersten 20 Jahre dominierten Portraitfotografien. ${ }^{20}$ Die frühen Fototechniken gaben Grüntöne nur unzureichend wieder, so dass die Landschaftsfotografie und mit ihr Fabrikfotografien erst in den 186oer Jahren populär wurden. ${ }^{21}$ Zusätzlich kamen erste zu dieser Zeit kleinere, tragbare Fotokameras auf den Markt, die es Lichtbildnern erleichterten, Motive außerhalb ihrer Fotoateliers aufzunehmen. ${ }^{22}$ Jetzt konnten sie auch Aufträge auf Fabrikgeländen annehmen.

Die Beziehung zwischen Auftraggebern und Fotografen variierte je nach Unternehmen. Vereinzelt ließen westeuropäische und amerikanische Firmenleitungen im 19. Jahrhundert hauseigene Fotoateliers einrichten, was in diesen Fällen zu einer großen Anzahl an Negativen und einer enormen Bandbreite an Motiven im Firmennachlass beitrug. ${ }^{23}$ Einige russische Firmen richteten im 19. Jahrhundert schon eigene Reklameabteilungen ein, so beispielsweise die Parfüm- und Seifenfabrik Brokar i Ko aus Moskau. ${ }^{24}$ Es gibt keine Hinweise

Entwicklung der Industriefotografie, in: Alfried Krupp von Bohlen und Halbach-Stiftung (Hrsg.): Krupp. Fotografien aus zwei Jahrhunderten, Berlin, München 2011, S. 42-55, S. 4243; Ulrich Pohlmann: Obelisken der Industrie - Poesie der Technik. Anmerkungen zur Industriefotografie im 19. Jahrhundert, in: ders.; Rudolf Scheutle (Hrsg.): IndustrieZeit. Fotografien 1845 - 2010, Tübingen 2011, S. 7-10, S. 8; Pugh: Industrial Image, S. 10-11; Pohlmann: Industriebilder, S. 236.

19 Zu Deutschland siehe beispielsweise: Matz, Industriefotografie, S. 29; zu Frankreich: Assegond: La photographie du travail, S. 163; zu Großbritannien: Pugh: Industrial Image, S. 8-9; zu den Niederlanden: Mattie Boom: Moderne Landschaft. Die Ingenieurfotografie und das Bild der Niederlande, in: Jenny Reynaerts (Hrsg.): Der weite Blick. Landschaften der Haager Schule aus dem Rijksmuseum, München 2008, S. 69-97, S. 75; zu Russland: MIZ: Al'bom bol'šoj: o. A.: Admiralitejskij ižorskij zavod, o. O. 1866.

20 Elliot: The Photograph in Russia, S. 13.

21 Jäger: Gesellschaft und Photographie, S. 206; Ulrich Pohlmann: Naturwunder und Territorium. Anmerkungen zur Landschaftsfotografie im 19. Jahrhundert, in: Fotogeschichte. Beiträge zur Geschichte und Ästhetik der Fotografie, Jg. 31/2011, Heft 120, S. $5^{-20}$, S. 7 .

22 Elena Barchatova: Realism and Document: Photography as Fact, in: David Elliot (Hrsg.): Photography in Russia 1840-1940, London 1992, S. 41-50, S. 41.

23 Das erste entsprechende Beispiel ist die deutsche Firma Krupp. von Dewitz: Die Bilder sind nicht teuer, S. 41. 1897 gründete General Electrics ein eigenes Werbebüro, zu dem auch Fotografen gehörten. Nye: Image Worlds, S. 14-15. 1898 stellte auch die AEG einen Fotografen an. Henning Rogge: Fabrikwelt um die Jahrhundertwende am Beispiel der AEG Maschinenfabrik in Berlin-Wedding, Köln 1983, S. 22. In Frankreich stellte die Firma Renault 1911 einen eigenen Fotografen ein, um ihre Werke und Produktion mittels Fotografien und Fotoalben zu klassifizieren. Assegond: La photographie du travail, S. 203.

West: I Shop in Moscow, S. 54. 
darauf, dass Angestellte selbst fotografierten. Üblicherweise wurden die Aufnahmen nicht von Angestellten der Betriebe gemacht, sondern Unternehmer vergaben Aufträge an externe professionelle Fotografen. Amateurfotografien aus dem Kontext der Fabriken gibt es so gut wie keine. ${ }^{25}$

Häufig hatten die Beauftragten keine besondere Erfahrung auf dem Gebiet der Industriefotografie. Während sich Fotografen in Bereichen wie Portraitund Landschaftsfotografie oder Genrebildern zu Spezialisten entwickelten, ${ }^{26}$ war es die Ausnahme, dass sich Fotografen vor dem Ersten Weltkrieg auf Motive aus Fabriken spezialisierten. ${ }^{27} \mathrm{Im}$ russischen Kontext ist kein solcher Fall bekannt. Dies hängt auch mit der wirtschaftlichen Struktur des Zarenreichs zusammen, die bis über den Ersten Weltkrieg hinaus in erster Linie landwirtschaftlich geprägt war. Die im europäischen Vergleich eher geringe Anzahl an industriellen Großbetrieben reichte vermutlich nicht aus, um die Existenz spezialisierter Industriefotografen sicherzustellen.

Aufnahmen von Fabriken und Industrieanlagen waren darüber hinaus keine Motive, mit denen Fotografen sich besonderes Ansehen innerhalb ihrer eigenen Zunft erarbeiten konnten. Dies spiegeln Sektionen und Exponate fotografischer Ausstellungen, Industriefotografien spielten dort kaum eine Rolle. ${ }^{28}$ 1911 organisierte die kaiserliche Akademie der Künste in der russischen Hauptstadt die allrussische Ausstellung zur künstlerischen Architekturfotografie. Der zugehörige Katalog listet die Namen der Fotografen und die Titel ihrer ausgestellten Fotografien auf. Unter den 1.014 aufgeführten Exponaten zeigte

$25 \mathrm{Zu}$ nennen wären hier höchstens die Fotografien eines Ingenieurs der Ižorskie zavody. Siehe Reproduktionen im MIZ.

26 Portraitfotografien waren das am weitesten verbreitete Genre in der Frühzeit der Fotografie im Zarenreich. Elliot: The Photograph in Russia, S. 13. Evgenij Petrovič Višnjakov (1841-1916) gilt als erster russischer Landschaftsfotograf. Vladimir Andreevič Karrik (18271878) gilt als Begründer der russischen Genrefotografie. Elena V. Barchatova: Wissenschaft? Handwerk? Kunst!, S. 13, 18; Vil'jam Karrik: Kartiny russkoj žizni, Sankt-Peterburg 2010.

27 In Frankreich war Gabriel Gorce (1863-?) eine solche Ausnahme. Gorce spezialisiert sich auf die Herstellung von Fotoalben, die große Fabriken für ein breites Publikum zugänglich machten. Céline Assegond: Les débuts de la photographie du travail usinier. Production, représentation usages (1870-1915), in: Jean-Paul Géhin; Hélène Stevens (Hrsg.): Images du travail, travail des images, Rennes 2012, S. 87-100, S. 94.

28 In Moskau gab es auf der Fotografieausstellung 1889 folgende Abteilungen: I. Historische Fotografie; II. Instrumente, Materialien; III. Portraits und Gruppenaufnahmen; IV. Fotografien außerhalb des Pavillons; V. Wissenschaftliche Verwendung von Fotografie; VI. Fotomechanische Prozesse. Barchatova: Russkaja svetopis', S. 233. 
dem Titel nach nur eine Aufnahme einer Fabrik. ${ }^{29}$ Abzüge von Kirchen und Klöstern dominierten die ausgestellten Motive.

Ebenso verdeutlichen die Inhalte zeitgenössischer fotografischer Zeitschriften, dass es sich bei Aufnahmen industrieller Motive um ein Randgebiet des fotografischen Gewerbes handelte. ${ }^{30}$ Die Zeitschriften berichteten über technische Neuentwicklungen, über fotografische Gesellschaften und Ausstellungen, die Kunstfotografie, die praktische Anwendung der Aufnahmen beispielsweise in der Wissenschaft sowie über Experimente zur Farbfotografie. ${ }^{31}$ Stichprobenartige Untersuchungen von fünf russischen Zeitschriften zwischen 1885 und 1909 ergaben nur einen einzigen Artikel, der sich näher mit der Fotografie von Maschinen beschäftigte. ${ }^{32}$

Der Autor des Artikels betonte, dass die Fotografien von Maschinen nicht nur die eigene Produktpalette erweitere, sondern dass Aufträge aus der Industrie ein lukratives Geschäft seien..$^{33} 1910$ ging bei der Maschinenfabrik in Kolomna ein Kostenvoranschlag des Unternehmens Frišmut i Marks aus St. Petersburg über 169 Rubel für die Herstellung von neun Fotografien ein eine hohe Summe. ${ }^{34}$ Zum Vergleich verdiente ein Arbeiter der St. Petersburger Gummiwarenfabrik Treugol'nik 1909 im Monat durchschnittlich 33 Rubel. ${ }^{35}$ Angesichts des teilweise großen Aufwands relativiert sich die hohe Summe jedoch. Lichtbildner mussten häufig weite Anreisen bis zu ihrem Arbeitsort

29 Imperatorskaja Akademija Chudožestv (Hrsg.): Katalog 1-oj vserossijskoj vystavki chudožestvenno-architekturnych fotografii. Ustroennoj obščestvom architektorovchudožnikov odnovremenno s IV sezdom russkich zodčich, Sankt-Peterburg 1911, S. 80.

30 Dies gilt auch für Frankreich, wo fotografische Zeitschriften ebenfalls nur selten über Fotografien mit industriellen Motiven berichteten, während sie zur Verwendung von Fotografien in der Astronomie, Archäologie oder in anderen Bereichen der Wissenschaften regelmäßig Artikel publizierten. Assegond: La photographie du travail, S. 45. $\mathrm{Zu}$ fotografischen Zeitschriften in Russland: Barchatova: Wissenschaft? Handwerk? Kunst!, S. 20-31.

32 o. A.: Fotografirovanie mašin, in: Fotografičeskoe Obozrenie, Jg.5/1899, Heft 11, S. 422-424. Folgenden Zeitschriften wurden, nach Themen mit Bezug zu Fabrikfotografien durchgesehen: Fotografičeskij Listoček (Das fotografische Blättchen), Nov. 1906-Dez. 1907; Fotograf Ljubitel' (Der Amateurfotograf), 189o; Fotografičeskie Novosti (Fotografische Neuigkeiten), Feb.-März 1907, Jan. 1908, Dez. 1909; Fotografičeskoe Obozrenie (Die fotografische Rundschau), Nov. 1885-Okt. 1887; Fotografičeskij Vestnik (Der fotografische Bote), 1887-1888, 1899 .

33 o. A.: Fotografirovanie mašin, S. 422. Dies gilt auch für Deutschland: Andreas Zeising: Dramatik und Distanz. Positionen der Industriefotografie im 20. Jahrhundert, in: Sabine Beneke; Hans Ottomeyer (Hrsg.): Die zweite Schöpfung. Bilder der industriellen Welt vom 18. Jahrhundert bis in die Gegenwart, Wolfratshausen 2002, S. 114-119, S. 114.

34 CGA Moskvy f. 318, op. I t 1, d. 672, l. 221.

35 CGIA f. 1179, op. 27, d. 1, 1. 167. 
in einer Fabrik unternehmen. ${ }^{36}$ Dort angekommen beeinflussten äußere Umstände die Herstellung von Industriefotografien, die sich deutlich aufwendiger gestaltete als Portraitaufnahmen im eigenen Studio. Der französische Fotograf Henri Émile Cimarosa Godefroy (1837-1913) beschrieb die Schwierigkeiten, denen er sich ausgesetzt sah, nachdem er einen Auftrag für acht Außenaufnahmen der Forges de Montataire (Oise) übernommen hatte. Während er einerseits vom Wetter abhängig war, konnte er nur an arbeitsfreien Tagen, die es alle drei Wochen gab, fotografieren. An Arbeitstagen ließ der Rauch der Schornsteine das Motiv unscharf werden. Damit nicht genug: Godefroy konnte nur in den Wintermonaten fotografieren, weil sonst das Laub der Bäume den Blick auf die Fabrik verdeckte. Insgesamt musste Godefroy fast ein Dutzend Mal anreisen, bis er die gewünschten Aufnahmen vorweisen konnte. ${ }^{37}$ Dies relativiert den vergleichsweise hoch anmutenden Preis für Fabrikfotografien.

Nichtsdestotrotz handelte es sich um ein lukratives Geschäft. Dies bewog in Russland viele Fotografen wie Karl Karlovič Bulla (1855-1930), in ihren Anzeigen damit zu werben, dass Mitarbeiter ihrer Ateliers bereit seien, Auftragsarbeiten außerhalb der Hauptstadt anzunehmen. ${ }^{38}$ Auch Fotografen in Rostov na Donu und Ekaterinburg nutzten Werbeanzeigen, um Industrielle auf sich aufmerksam zu machen und zusätzliche Aufträge zu erhalten. ${ }^{39}$

Meist bedurfte es eines besonderen Anlasses, damit Fabrikanten einen externen Fotografen anstellten, der wie Bulla aus der Hauptstadt anreisen musste. ${ }^{40}$ Üblicherweise sparten Unternehmen die Kosten für Fahrt und Unterkunft, indem sie ihre Aufträge an lokale Fotografen vergaben.$^{41}$ Leider lässt sich

$36 \quad$ Je nach Vertrag kam der Auftraggeber neben dem Lohn für die Arbeit des Fotografen auch für dessen Reisekosten und Unterkunft auf. Stolarski: The Rise of Photojournalism, S. 46.

37 Dabei musste er jedes Mal auch die gesamte Ausrüstung zum Aufnahmeort bringen. Assegond: La photographie du travail, S. 104, 115.

38 Vladimir Anatol'evič Nikitin: Rasskazy o fotografach i fotografijach, Leningrad 1991, S. 85. In Frankreich befand sich die Fotografie Anfang des 20. Jahrhunderts in einer Krise: Aufgrund der zunehmenden Anzahl an Fotografen erhielten nicht mehr alle automatisch genug Aufträge. Auch wenn es in der Literatur zur Situation im Zarenreich keine eindeutigen Hinweise auf eine vergleichbare Situation gibt, ist denkbar, dass dies mit ein Grund war, warum sich Fotografen selbst um Aufträge bemühten. Assegond: La photographie du travail, S. 105.

39 V. L. Metenkov: Ukazatel' cene na proizvedenija fotografičeskago atel'eV. L. Metenkovai Ko v Ekaterinburge, Ekaterinburg 1888, S. 19; L. F. Vološinova; A. P. Derkač: Rostov-na-Donu. Portret goroda na rubeže XIX-XX vekov, Rostov 2007, S. 26.

40 Ein Beispiel hierfür sind die Fotoalben von Ivan Grigro'evič D'jagovčenko (1835-1887): RGB FO: Inv MK XII-4224: Tul'skij Imperatora Petra Velikago oružejnyj zavod (Hrsg.): Tul'skij oružejnyj zavod. Osnovan 1712, perestroen 1873, o. O. 1873 .

41 So vergab die Waffenfabrik in Tula Aufträge an Vladimir Ivanovič Vakulenko (1868-1925?) und Semën Osipovič Kanter (unklar), und die Maschinenfabrik in Kolomna an die Brüder 
heute oft nur schwer nachvollziehen, wer Urheber einer Fotografie war, denn es war nicht üblich, mit dem Namen des Fotografen zu werben, selbst wenn es sich um einen renommierten Vertreter seines Fachs handelte. ${ }^{42}$ Deshalb bleiben die meisten Lichtbildner der überlieferten Fabrikaufnahmen anonym. Die Fotografen traten ihre Bildrechte und die entsprechenden Negative an ihre Auftraggeber ab, ${ }^{43}$ so dass sie nicht selbst mit diesen Arbeiten für sich werben konnten. ${ }^{44}$

\subsection{Fotografien im internen Gebrauch der Fabriken}

Am neuen Medium der Fotografie bewunderten die Zeitgenossen insbesondere deren Detailgenauigkeit, die die Malerei in dieser Exaktheit nicht erreichte. ${ }^{45}$ Darüber hinaus waren die neuen Bilder verglichen mit Ölgemälden deutlich preiswerter. ${ }^{46}$ Beide Charakteristika trugen dazu bei, dass die Fotografie schnell einen festen Platz in großen Fabriken erhielt und fotografische Aufnahmen in zahlreichen Kontexten zum Einsatz kamen. Briefe aus dem Firmenarchiv des Kolomenskij mašinostroitel'nyj zavod (Kolomnaer Maschinenbaubetrieb) ${ }^{47}$ belegen, dass Glasnegative und Abzüge für Fabrikbesitzer trotz ihres geringeren Preises wichtige und wertvolle Objekte waren. In mehreren Fällen wandten sich Angestellte des Unternehmens an Druckereien oder Hersteller von Druckplatten. Sie baten die Adressaten, alle Fotografien und Glasnegative, die sich noch von früheren Aufträgen und Bestellungen bei ihnen befänden, nach Kolomna zurückzuschicken. ${ }^{48}$ In manchen Fällen

Epifanov (unklar), siehe: Kapitel „Inszenierte Geschichte - Firmenjubiläen“, S. 179-240; Sergej Ivanovič Samošin: Putešestvie v staruju Kolomnu, Kolomna 2007, S. 26, 28.

Dies gilt für die Schokoladenfabrik Abrikosovyj und die Aufnahmen Levickijs (es ist allerdings unklar, ob es sich um Vasilij Vasil'evič oder um Andrej Andreevič handelt), ebenso für die Putilovwerke und die Fotografien Bullas. RGB: INV MK XII-1277: 0. A.: Vidy fabrik i magazinov Tovariščestva A. I. Abrikosova synovej, v 1888 g., Moskva 1888; CGAKFF: G 666; E 3418; E 3414; E 3417; E 3405; Ž 203.

43 Stolarski: The Rise of Photojournalism, S. 114.

44 Dies hat zur Folge, dass in Nachschlagewerken Fotografen nur für ihre Portraits gewürdigt werden, obwohl sie auch Industrieaufnahmen anfertigten. Siehe: Kapitel „Neue Bilder eines neuen Raums - Fabrikalben“, S. 103-178.

45 Jäger: Fotografie und Geschichte, S. 55.

46 Assegond: La photographie du travail, S. 202.

47 Die Brüder Gustav Struve (1834-1882) und Armand Struve (1835-1898) gründeten 1863 den Kolomenskij Mašinostroitel'nyj zavod, der zunächst Eisenbahnbrücken herstellte. Die Produktpalette erweiterte sich bald um beispielsweise Lokomotiven, Motoren und Dampfschiffe.

48 CGA Moskvy f. 318, op. I t 1, d. 672, l. 107, 138, 159. 
war es aufwendig und dauerte lange, bis alle Negative in die Firma zurückkamen und ein neuer Katalog gedruckt werden konnte. ${ }^{49}$ Der Wert guter Aufnahmen zeigte sich auch darin, dass Fabriken sie über mehrere Jahre hinweg in verschiedenen Kontexten verwendeten. ${ }^{50}$ Aufträge für neue Aufnahmen vergaben Unternehmen nur, wenn sie ein Negativ verloren hatten, die Vorlage durch die häufige Verwendung ihre Qualität eingebüßt hatte oder von einem neuen Motiv Bilder anzufertigen waren. ${ }^{51}$

Welche Motive von Bedeutung waren, hing von den Funktionen ab, die die Fotografien erfüllen sollten. Für die fabrikinterne Verwendung spielte der Aspekt der Dokumentation eine entscheidende Rolle. Russische Unternehmen nutzten, ähnlich wie in Westeuropa, Fotografien von Baustellen und Fabrikgebäuden, um voranschreitende Baumaßnahmen aufzuzeichnen oder um den Gebäudebestand des Unternehmens zu einem bestimmten Zeitpunkt festzuhalten.52 Vorbild dieser dokumentarischen Praktik waren die großen staatlichen Infrastrukturprojekte des 19. Jahrhunderts wie der Eisenbahnbau. Bereits 1847 ließ sich der Ingenieur Isambard Kingdom Brunel (1806-1859) wöchentlich Daguerreotypien der Bauarbeiten an der Eisenbahn zwischen Florenz und Pistoia schicken.53 Ähnliche Dokumentationen entstanden auch im Zarenreich, beispielsweise für die Eisenbahnstrecke St. Petersburg-Warschau. ${ }^{54}$

Fabriken nutzten das neue visuelle Medium darüber hinaus, um ihre Produktpalette festzuhalten. Zu diesem Zweck bestellten Industrielle Fotografien von Maschinen, Lokomotiven, Waggons oder Schiffen, je nach

49 CGA Moskvy f. 318, op. I t 1, d. 672, l. 284. Mit der Verwendung von Fotografien in Katalogen partizipierten die Unternehmen an einem europaweiten Trend. So hatten Fotobücher in Großbritannien in erster Linie die Funktion zu klassifizieren und einzuteilen, was in Katalogen ebenfalls geschieht. Auch Starl beschreibt den Prozess der Katalogisierung und der Illustrierung von Katalogen mit Fotografien als typisch für das 19. Jahrhundert. Martin Parr; Martin Badger: The Photobook. A History, Bd. 1, London u. a. 2004, S. 40; Starl: Im Prisma des Fortschritts, S. 15 .

5o Dies war beispielsweise bei einer Fotografie des Elektrizitätswerks der Maschinenfabrik in Kolomna der Fall. Die Fotografie erschien 1903 in einer Festschrift des Unternehmens, in einem undatierten Werbeprospekt der frühen 1910er Jahre und 1916 in einem Album. o. A.: Techničeskoe opisanie Kolomenskago mašinostroitel'nago zavoda 1863-1903 g.g. K vypusku 3000-go parovosa. Kolomna 1903, S. 18a; FS: Schachtel: 282a, Dokument 6 B5: o. A.: Kolomenskij Zavod: Dvigateli Dizel', o. O. o. J., S. 36; RNB: $\dot{\mathrm{E}}((\mathrm{Al}$ Tchıo $) /(2-2))$ : o. A.: Kolomenskij Mašinostroitel'nyj zavod, o. O. 1916, S. 1.

$51 \quad$ CGA Moskvy f. 318, op. I t 1, d. 672, l. 219; f. 318, op. 1, d. 746, l. 159 .

52 Führend waren in den 185 oer Jahren auf diesem Gebiet insbesondere französische Ingenieure. Keckeis: Fotografien im Dienste expansiver Unternehmensstrategien, S. 22-25.

53 Assegond: La photographie du travail, S. 56.

54 Pohlmann: Naturwunder und Territorium, S. 11. 
Geschäftszweig des Unternehmens. Diese Produktfotografien waren besonders in der metallverarbeitenden Industrie, vorzugsweise in Betrieben verbreitet, die im Maschinenbau (im weitesten Sinne) tätig waren. In Firmennachlässen der Nahrungsmittel- oder Textilindustrie finden sich so gut wie keine Produktfotografien. Hier reichte die Zweidimensionalität und begrenzte farbliche Wiedergabe der Fotografien nicht aus, um die wesentlichen Merkmale der Fabrikate festzuhalten (sei es der Geruch einer Seife oder die Farbgebung eines Stoffmusters). Selbst in der Waffenfabrik in Tula finden sich keine fotografischen Aufnahmen von einzelnen Gewehren - zur Dokumentation dienten technische Zeichnungen. Dafür finden sich sowohl im Museum der Maschinenfabrik in Kolomna als auch im Museum der Putilovwerke zahlreiche Abzüge von Lokomotiven, Schiffen oder sogar Brücken, die die Unternehmen hergestellt und gebaut hatten. In diesen Fällen hielten die Fotografien den Gesamteindruck fest, während Baupläne und technische Zeichnungen die Details dokumentierten. ${ }^{55}$

Außerdem fungierten Fotografien als Beweisstücke im Falle von Unglücken, wenn durch Brände oder Überschwemmungen Schäden an Fabrikgebäuden entstanden. ${ }^{56}$ Solche Aufnahmen finden sich vor allem in Archiven von Unternehmen, bei denen es sich um Niederlassungen ausländischer Firmen handelte. Denkbar wäre, dass russische Betriebe keine solchen Fotografien aufnahmen. Wahrscheinlicher scheint jedoch, dass unterschiedliche Archivierungspraktiken dazu führten, dass heute entsprechende Aufnahmen besonders von Auslandsniederlassungen existieren. Anlässlich von Schadensfällen sandten die russischen Vertreter der Farbenfabriken Friedrich Bayer \& Co oder des Chemieproduzenten Geigy Fotografien an die jeweiligen Mutterunternehmen nach Deutschland beziehungsweise in die Schweiz. ${ }^{57}$ Die Niederlassung der Farbenfabriken Friedrich Bayer \& Co hatte im April 1908 große Hochwasserschäden zu beklagen. Die Firmenleitung identifizierte die Betreiber der Moskauer Rundbahn als Verantwortliche für das Unglück, weil der Bahndamm verhinderte, dass das Wasser abfloss, und stattdessen die

55 Zur Entwicklung der technischen Zeichnung siehe: Ken Baynes; Francis Pugh: The Art of the Engineer, Guildford 1981. Siehe auch: o. A.: Al'bom vidov i sooruženij železnoj dorogi ot Tiflisa do Persidskoj granicy, Džul'fy s vetvjami k gor. Karsu i Ėrivan. 1895-1907, Tiflis 1909, S. 7, 15, 29, 32-33.

$5^{6}$ Sie wurden auch als Beweis und zur Untermauerung von Rechenschaftsberichten auf Generalversammlungen an Aktionäre verteilt. Keckeis: Fotografien im Dienste expansiver Unternehmensstrategien, S. 42.

57 In folgenden Firmenarchiven sind Fotografien von durch Hochwasser überfluteten Fabrikgeländen archiviert: Geigy: KAR 1; BU: Objekt-Nr. 3437-3442; oder durch Feuer beschädigter Gebäude: MIZ: zwei unsortierte Fotografien. 
Strömung erhöht hatte. ${ }^{58}$ Mit großer Wahrscheinlichkeit dienten die Fotografien nicht nur dem Rapport nach Deutschland, sondern auch als Beweismaterialien im anschließenden Gerichtsprozess. Innerhalb Deutschlands und Großbritanniens kamen Fotografien häufig im Falle juristischer Streitigkeiten zum Einsatz, oder um Schadensmeldungen an Versicherungen zu machen. ${ }^{59}$

Eine weitere Funktion übernahmen Fotografien in russischen wie westeuropäischen Fabriken in Ausbildung und Lehre. Autoren russischer fotografischer Zeitschriften betonten immer wieder, welche Bedeutung den neuen Bildern innerhalb des Schulunterrichts zukomme, auch wenn bis dahin nur wenige Schulen in Russland dieses Potential genutzt hätten. ${ }^{60}$ Fotografien könnten die Konzentration der Schüler fördern ${ }^{61}$ und gerade Stereoskopien vermittelten ihnen einen besonders lebendigen Eindruck und ließen sie virtuell in jedes beliebige Land reisen. ${ }^{62}$ Es scheint wahrscheinlich, dass auch Fabriken, angeregt durch diese und ähnliche Debatten, die neuen Bilder als pädagogisches Anschauungsmaterial für die Ausbildung ihrer Arbeiter einsetzen. ${ }^{63}$ So findet sich in einem Album der Kettenfabrik L. L. Zotov in Nižnij Novgorod die Fotografie einer Schautafel, auf der Aufnahmen von geborstenen Kettengliedern zu sehen sind. Eine zweite Aufnahme zeigt eine weitere Tafel mit zuvor geborstenen Kettengliedern, die Arbeiter der Fabrik wieder repariert hatten. ${ }^{64}$ Die beiden Abbildungen zeigten sowohl Mängel

$5^{8}$ A. Blank; W. Löw: Geschichte der Fabrik der Farbenfabriken vorm. Friedrich Bayer \& Co in Moskau, o. O. 1914, S. 280-281.

59 Lüdtke: Industriebilder - Bilder der Industriearbeit?, S. 403; Pohlmann: Obelisken der Industrie - Poesie der Technik, S. 9. Leider finden sich weder in den Findbüchern des CGA Moskvy noch des CGIA Hinweise, dass es in den Akten von Versicherungsunternehmen entsprechende Fotografien gegeben haben könnte. Auch eine stichprobenartige Analyse der „Moskovskoe strachovoe ognja obščestvo“ gab keine Anzeichen vergleichbarer Aufnahmen. CGA Moskvy f. 299, op. 2, d.66o; f. 299, op. 2, d. 1072. Allerdings kann dies möglicherweise auch damit zusammenhängen, dass die Versicherungen oder Archive die Fotografien vor der Archivierung aussortierten.

60 o. A.: Školnaja zanjaija fotografiej, in: Fotografičeskie Novosti, 1909, Heft 12, S. 181-182, S. 181. Eine Bildungseinrichtung, die Lichtbilder zu pädagogischen Zwecken einsetzte, war die St. Petersburger Militärakademie. Im Geschichtsunterricht verwendeten Lehrer hier bereits in den 186oer Jahren fotografische Projektionen, um die Lehrinhalte anschaulicher zu gestalten. Jens Ruchatz: Licht und Wahrheit. Eine Mediumgeschichte der fotografischen Projektion, München 2003, S. 212-213.

61 o. A.: Neskol'ko slov pedagogam, in: Fotografičeskie Novosti, 1907, Heft 2, S. 17-19, S. 17.

62 o. A.: Stereoskop kak učebnoe posobie, in: Fotografičeskie Novosti, 1909, Heft 9, S. 129-130, S. 129 .

63 Zu Deutschland beispielsweise Lüdtke: Industriebilder - Bilder der Industriearbeit?, S. 406.

64 RGB: Inv MS XIII-4241: o. A.: Vidy cepodelatel'nago zavoda i pervoj v Rossii meždunarodnoj ispytatel'noj stancii v Nižnjago-Novgoroda L. L. Zotova (nasl. A. A. Smelova), Nižnij Novgorod o. J., S. 11-12. 
in der Produktion als auch Techniken, diese zu beheben. Fotografische Aufnahmen hielten auch Arbeitsunfälle fest, so dass Auszubildende im Nachhinein nachvollziehen konnten, was passiert war und wie dies hätte verhindert werden können. ${ }^{65}$

Neben unbelebten Motiven hatten auch Aufnahmen von Menschen einen hohen Stellenwert innerhalb russischer Fabriken. Portraits waren in Büroräumen, in Speisesälen oder Lehreinrichtungen als Wandschmuck angebracht. ${ }^{66}$ Die Eigenschaft der Fotografien, die Roland Barthes als Verschränkung des realen und des Lebendigen ${ }^{67}$ beschreibt, spielte hier eine entscheidende Rolle. Um eine Fotografie zu erhalten, müsse sich die entsprechende Person vor der Kamera befunden haben, was für eine besondere Form der Authentizität sorge. Gleichzeitig vermittle die Fotografie einerseits das Gefühl, die Person sei noch lebendig, auch wenn diese möglicherweise bereits gestorben sei. Andererseits sei jede Fotografie ein "memento mori“, denn sie erinnere an das unerbittliche Verfließen der Zeit - alles Abgebildete befinde sich automatisch in der Vergangenheit. ${ }^{68}$ Fabrikanten nutzten besonders Portraits wichtiger Persönlichkeiten aus der Unternehmensgeschichte oder Aufnahmen der Zaren, um eine virtuelle Präsenz dieser Menschen zu demonstrieren. ${ }^{69}$ Die Ähnlichkeit zwischen Abbild und Portraitiertem ließ letzteren als scheinbar unmittelbar anwesend erscheinen und schuf eine Verbindung zwischen Betrachter und Fotografiertem. Somit demonstrierten die Aufnahmen eine virtuelle Nähe des Betriebs zum Zaren oder sie dienten den Arbeitern als Identifikationsangebote, indem sie zumeist auf den Fabrikgründer als Vater der großen Unternehmensfamilie verwiesen.

Gruppenaufnahmen von Arbeitern konnten ebenfalls dazu beitragen, dass sich unter der Belegschaft ein Gemeinschaftsgefühl entwickelte. ${ }^{70}$ Häufig spiegeln diese Fotografien die firmeninterne Einteilung der Arbeiter in einzelnen Werkstätten:71 Das Individuum erhielt einen Platz vor der Kamera, weil

65 Lüdtke: Industriebilder - Bilder der Industriearbeit?, S. 406.

66 GPIB: LBI (4/159): o. A.: 1864 Zolotoj jubilej parfumernago proizvodstva tovariščestva Brokar i Ko v Moskve. 1914, Moskva 1914, S. 23; RNB OE்: Ė ((AlTch45o)/(1-1a)): o. A.: Tovariščestvo Krasnosel'skoj pisučebymažnoj fabriki naslednikov K. P. Pečatkina, Sankt-Peterburg (nach) 1911, S. 52; RNB OE்: $\dot{\mathrm{E}}((\operatorname{AlTch} 459) /(2-1))$ : o. A.: Tovariščestvo manufkatury Ivan Garelin i Synov'ja, Moskva o. J., S. 85.

67 Roland Barthes: Die helle Kammer. Bemerkungen zur Photographie, Frankfurt a. M. 1989, S. 93 .

68 Dazu auch: Sontag, In Platos Höhle, S. 280, 291.

69 Müller: Grundlagen der visuellen Kommunikation, S. 83.

70 Matz: Industriefotografie, S. 53.

71 Beispielsweise: GATO f. 3097, op. 3, d. 32, 84-133; RGB: IZO MK XII-1279: o. A.: Al'bom Tovariščestva sitcevoj manufaktury Al'berta Gjubner v Moskve, Moskva o. J, S. 5-15. 
es ein Teil dieser Werkstatt und folglich ein Teil des Unternehmens war. In anderen Fällen war es eine bestimmte Gruppe wie der Chor oder die Wohltätigkeitsgesellschaft, die vor der Kamera standen. In diesem Fall bestimmten individuelle Interessen die Zusammenstellung der Gruppenaufnahmen, ${ }^{72}$ doch auch hier bildete der Betrieb den Rahmen. Unter den Motiven der Industriefotografie sind Gruppenaufnahmen diejenigen Bilder, auf denen sich trotz der strikten Inszenierung durch den Fotografen immer wieder Hinweise auf zwischenmenschliche Beziehungen finden. Gruppenportraits zeigen oft Personen, die anderen eine Hand auf die Schulter legen, oder zwei Abgebildete halten sich an den Händen. ${ }^{73}$ Diese Gesten waren mit großer Wahrscheinlichkeit nicht intendiert. Weil es sich jedoch häufig um Fotografien zur internen Verwendung handelte, störte sich die Fabrikleitung nicht an ihnen und verzichtete auf Retuschen.

Fotografien veränderten weiter die Arbeitsabläufe in den Fabriken. Frederick Taylor (1856-1915) bediente sich der neuen visuellen Technik für seine Studien zur Messung und Überwachung industrieller Arbeit. ${ }^{74}$ Er analysierte einzelne Arbeitsschritte und Bewegungen, um sie in der Folge zu standardisieren. Hierzu nutzte er die Möglichkeit der Fotografie, schnell aufeinanderfolgende Aufnahmen zu machen und auf diese Weise eine Bewegung in eine Serie von Standbildern aufzuspalten. ${ }^{75}$ Es ist denkbar, dass auch im Zarenreich Fotografien für vergleichbare Projekte zum Einsatz kamen. Allerdings geben die im Rahmen der Arbeit analysierten Firmenarchive keine Hinweise darauf.

\subsection{Fotografien in der externen Kommunikation der Fabriken}

Die Fotografie rief auch neue Entwicklungen in der externen Kommunikation der Unternehmen hervor. Sie spielte vor allem im Bereich der Reklame eine entscheidende Rolle. Die neuen visuellen Aufnahmen ermöglichten es Industriellen, ihre langjährige Erfahrung in der Werbetätigkeit und öffentlichen Selbstdarstellung auf eine neue Ebene zu heben. Für das Zarenreich wird der

\footnotetext{
72 Chor: o. A.: 1857-1907 Krengol'mskaja manufaktura. Istoričeskoe opisanie. Sostavlennoe po slučaju 5o-ti letija eji suščestvovanija, ispolnivšagosja 30 aprel’ja 1907 goda, SanktPeterburg 1917, Anhang; Wohltätigkeitsgesellschaft: MIZ: unsortierte Fotografie.

73 Beispielsweise: GATO f. 3097, op. 3, d. 84.

74 Elspeth H. Brown: The Corporate Eye. Photography and the Rationalization of American Commercial Culture, 1884-1929, Baltimore, London 2005, S. 6-7. Auch in Frankreich fanden Fotografien bei der Überwachung von Arbeitern Verwendung. Rouillé: Les images photographiques du monde du travail, S. 40-41.

Brown: The Corporate Eye, S. 68-73.
} 
Beginn unternehmerischer Reklametätigkeit auf das 18. Jahrhundert datiert. Bereits unter Peter I. nutzten russische Unternehmer markante Zeichen, ${ }^{76}$ um vergleichbar mit einer Frühform der Markenembleme ${ }^{77}$ bei den Kunden Assoziationen zwischen Produkten und der eigenen Firma zu wecken. Die erste Darstellung einer russischen Fabrik in diesem Kontext stammt aus den 1730er bis 1740er Jahren von der Verpackung einer Papierfabrik und zeigt eine stark vereinfachte Darstellung der Papierherstellung in der Fabrik F. Turuntaevskogo. ${ }^{78}$ Ende des 18. Jahrhunderts begann das Ladenschild seinen Siegeszug durch russische Städte und erhielt ab der zweiten Hälfte des 19. Jahrhunderts Gesellschaft von gedruckten Werbeplakaten. ${ }^{79}$ Bis zum Beginn des Ersten Weltkriegs waren Plakate in der Provinz das bevorzugte Werbemedium. ${ }^{80}$ Insgesamt nahm die Reklametätigkeit der Unternehmen insbesondere in den letzten Jahrzehnten des 19. Jahrhunderts deutlich an Fahrt auf. Als Grund hierfür nannten bereits Zeitgenossen den zunehmenden Konkurrenzdruck und den damit einhergehenden Kampf um die Aufmerksamkeit der Käufer. ${ }^{81}$

Die Werbethematik verdeutlicht das Nebeneinander von Hoffnung und Angst der Zeitgenossen angesichts neuer gesellschaftlicher Entwicklungen im 19. Jahrhundert. ${ }^{82}$ Im Kontext dieser Spannung investierten nicht alle Fabrikanten in gleicher Weise in Werbung: Sowohl unter den Unternehmern als auch unter den Publizisten gab es Stimmen, die der Firmenreklame skeptisch gegenüberstanden. Lutz Häfner sieht als eine Ursache die latent negative Haltung der russischen Gesellschaft gegenüber Kaufleuten und Handel generell. Werbung sei in diesem Kontext als Betrugsversuch verstanden worden. ${ }^{83}$ Diese Einschätzung deckt sich mit zeitgenössischen Beschreibungen. Trotz ihrer skeptischen Grundhaltung gaben die Autoren zu

76 Glinternik: Reklama v Rossii, S. 13.

77 A. M. Čebotarëv: Reformy Petra I i vozniknovenie Rossijskoj reklamy, Čeljabinsk 2005, S. 27.

78 Glinternik: Reklama v Rossii, S. 23.

79 West: I Shop in Moscow, S. 22, 28.

8 V V. V. Chojnovskij (Hrsg.): Uspešnaja reklama i kak' eju pol'zovat'sja, Kiev 1913, S. 39.

81 Nikolaj N. Plinskij: Reklama. Eë značenie, proischoždenie i istorija, Sankt-Peterburg 1894, S. 46; A. Ratner: Technika reklamy ob”javlenijami. Otdel'nyj ottisk iz „Roždestvenskago ukazatelja“ 1908 g. Torgovago Doma L. i. Ė. Metel' i Ko, Sankt-Peterburg 1909, S. 5; S. Ja.: Ob”javlenija i drugie sredstva reklamy. Zadači reklamy, sostavlenie i rasprostranenie različnych vidov eë, Moskva 1904, S. 3. Auch Verlage nutzten Reklame, um auf Neuerscheinungen aufmerksam zu machen und Rezensenten für ihre Publikationen zu finden. A. A. Bachtiarov: Slugi pečati. Očerki knigopečatnago dela, Sankt-Peterburg 1893, S. 192-193; Glinternik: Reklama v Rossii, S. 54.

82 West: I Shop in Moscow, S. 8.

83 Lutz Häfner: Medienpräsenz. Reklame im ausgehenden Zarenreich, in: Jörg Gebhard; Rainer Lindner; Bianka Pietrow-Ennker (Hrsg.): Unternehmer im Russischen Reich. 
bedenken, dass nicht die Präsentation eines Themas in unterschiedlichen Medien an sich über die Beurteilung der Werbung entscheide, sondern das Ziel, welches damit verfolgt würde. Dieses bestimme über den Wert der Reklame. ${ }^{84}$ Bei Werbung handle es sich, den Autoren zufolge, um eine Entwicklung, die besonders in den USA, England und Frankreich verbreitet sei, weswegen sie sich nicht ohne Weiteres auf die russische Leserschaft übertragen lasse. Es müsse zunächst eine spezifisch russische Form der Werbung gefunden werden. Diese sollte sich dadurch auszeichnen, dass sie die Leser informiere, ohne sie überzeugen zu wollen, so dass der Rezipient aufgrund der Lektüre einer Annonce eine fundiertere Entscheidung treffen könne. ${ }^{85}$ Diese Autoren lehnten reißerische Werbetexte und typografische Effekthascherei, wie Reklameexperten sie propagierten, ${ }^{86}$ dezidiert ab. Auch im Werbebereich spiegelte sich die im Zarenreich weit verbreitete Suche der Zeitgenossen nach dem genuin Russischen ihrer Kultur und die Auseinandersetzungen darüber, wie mit westeuropäischen Einflüssen umgegangen werden sollte.

Warum Unternehmen Werbung ablehnten, lässt sich im Einzelfall nicht nachvollziehen. Insgesamt war unter den Industriellen umstritten, wie groß die Erfolge beispielsweise von Annoncen tatsächlich waren. Zwar konnten sich Betriebsleitungen an der Auflagenzahl von Zeitungen und Illustrierten sowie an deren anvisierter Leserschaft orientieren, ${ }^{87}$ es blieb jedoch kompliziert nachzuvollziehen, wie das Publikum eine Anzeige wahrnahm. Diese Schwierigkeit nennt auch Sally West als Grund für eine mögliche Ablehnung seitens der Unternehmer. ${ }^{88}$ Als Reaktion gaben Reklameexperten Hinweise, wie Betriebe versuchen konnten, die Auswirkungen ihrer Werbeinvestitionen zu messen. ${ }^{89}$ Neben der Haltung der Firmenbesitzer war auch die finanzielle Situation eines Unternehmens entscheidend; Kleinbetriebe konnten beziehungsweise wollten sich entsprechende Ausgaben nicht leisten. ${ }^{90}$

Manche Wissenschaftler bezeichnen fotografische Aufnahmen in der zweiten Hälfte des 19. Jahrhunderts als das dominierende Medium visueller Firmenwerbung, ${ }^{91}$ eine Aussage, die für das Zarenreich differenziert werden

Sozialprofil, Symbolwelten, Integrationsstrategien im 19. und frühen 20. Jahrhundert, Osnabrück 2006, S. 265-292, S. 265.

84 Plinskij: Reklama, S. 17; Aleksej Verigin: Russkaja Reklama, Sankt-Peterburg 1898, S. 4.

85 Verigin: Russkaja Reklama, S. 8, 14-15.

86 Beispielsweise: Chojnovskij (Hrsg.): Uspešnaja reklama, S. 7.

87 Ratner: Technika reklamy, S. 8.

88 West: I Shop in Moscow, S. 44, 48.

89 Ratner: Technika reklamy, S. 15.

9o Anzeigen in Zeitschriften konnten je nach Auflagenzahl des Blattes sehr teuer werden. S. Ja: Ob"javlenija i drugie sredstva reklamy, S. 58-70.

91 Pugh: Industrial Image, S. 9; Nye: Image Worlds, S. 14-15. 
muss. Besonders für Fabriken, die Konsumprodukte wie Zigaretten oder Süßwaren herstellten, traf dies eher nicht zu. Stattdessen waren Plakate, Druckgrafiken oder bedruckte Verpackungen in diesen Industriezweigen die dominierenden Werbemedien. ${ }^{92}$ Allerdings ist zutreffend, dass visuelle Elemente in der Reklame zunehmend an Bedeutung gewannen. Dies spiegelt sich in vielen Publikationen, deren Autoren speziell darauf eingingen, dass Bilder die Aufmerksamkeit des Betrachters in besonderer Weise auf sich zögen. ${ }^{93}$ Die Illustrationen müssten aber von besonders guter Qualität sein, um den gewünschten Effekt zu erzielen. ${ }^{94}$

Auch neuere Forschungen teilen die Ansicht, dass sich die Verarbeitung von Bildern im menschlichen Gehirn maßgeblich von der schriftlicher Dokumente unterscheidet. Während Schrift erst entziffert werden muss, ist die Verarbeitung von Bildelementen schneller möglich und findet auf mehreren Ebenen statt wie Darstellungsform, Textur oder Inhalt. ${ }^{95}$ Regula Burri spricht von einer Gleichzeitigkeit der Wahrnehmung. ${ }^{96}$ Hinzu kommt, wie Marion Müller betont, die assoziative Kraft der Darstellungen und gerade bei Fotografien das Potential, Raum und Zeit zu überwinden und dadurch eine vermeintliche Unmittelbarkeit herzustellen, in der eine besondere Faszination liege. ${ }^{97}$

Um die Jahrhundertwende war im Zarenreich die russische Niederlassung der Firma Siemens einer der Vorreiter bei der Verwendung von gedruckten Fotografien in der Werbung. Siemens setzte als eines der ersten Unternehmen Autotypien für Reklamezwecke in russischen Zeitschriften ein. ${ }^{98}$ Das Unternehmen publizierte bereits 1899 Werbeanzeigen, die mit gedruckten

92 Glinternik: Reklama v Rossii, S. 41, 43; Häfner: Medienpräsenz, S. 268-269.

93 Beispielsweise: o. A.: Iskusstvo reklamirovat'. Prakt. Rukovodstvo dlja sostavlenija ob"javlenij, Odessa 1911, S. 16.

94 Chojnovskij (Hrsg.): Uspešnaja reklama, S. 13.

95 Türk: Bilder der Arbeit, S. 10.

96 Regula Valérie Burri: Bilder als soziale Praxis. Grundlegungen einer Soziologie des Visuellen, in: Zeitschrift für Soziologie, Jg. 37/2008, Heft 4, S. 342-358, S. 248. Ähnlich hierzu auch: Türk: Vorbemerkungen, S. 9. Hierzu auch: Josef Kasper: Belichtung und Wahrheit. Bildreportagen von der Gartenlaube bis zum Stern, Frankfurt a. M., New York 1979, S. 12.

97 Müller: Grundlagen der visuellen Kommunikation, S. 83.

98 Hier zeigt sich, dass lokale Niederlassungen der Firma eine relativ große Unabhängigkeit hatten. Firmengründer Werner von Siemens (1816-1892) war Werbung und Reklame gegenüber insgesamt eher skeptisch eingestellt und verfolgte stattdessen die Linie, dass das Unternehmen durch seine innovative Produktion beeindrucken solle. An diese Vorgabe hielt sich das deutsche Unternehmen auch lange Zeit im Vergleich zum Tochterunternehmen im Zarenreich. URL: https://www.siemens.com/history/de/ aktuelles/1186_warenzeichen.htm (zuletzt eingesehen am 24.08.15). 
Fotografien illustriert waren, ${ }^{99}$ obwohl illustrierte Zeitschriften im Zarenreich zu dieser Zeit erst begannen, Fotografien abzudrucken. ${ }^{100}$

Besonders in Fabriken, die nicht für den Massenkonsum produzierten wie Unternehmen der metallverarbeitenden Industrie, spielten Fotografien schon früh eine wichtige Rolle für die Präsentation des Betriebs. Das Zielpublikum dieser exklusiven Form von Werbung waren die Geschäftspartner, die von den Unternehmern gerahmte Abzüge fotografischer Aufnahmen als Geschenke erhielten. ${ }^{101}$

Originalabzüge hatten zudem eine wichtige Funktion, wenn Kunden Anfragen zum Kauf oder zu technischen Details von Produkten stellten. Im Archiv der Maschinenfabrik in Kolomna befinden sich in einer Reihe von Akten, die Anfragen für den Kauf von Maschinen aus der Produktion des Unternehmens enthalten, neben Details zu technischen Daten des jeweiligen Produkts auch Fotografien. Meist handelte es sich um klassische Produktfotografien, in diesem Fall von Maschinen oder Schiffen. ${ }^{102}$ Die Fotografien ergänzten die technischen Details eines Fabrikats wie Größe, Gewicht oder Leistungsstärke und ermöglichten dem Empfänger, sich ein Bild des Objekts zu machen und sich dadurch einen umfassenderen Eindruck des Produkts zu verschaffen. Gleichzeitig signalisierte die Firma ihren Partnern, wie sehr sie diese schätze, indem sie ihnen vergleichsweise hochpreisige Abbildungen zukommen ließ, noch bevor zwischen beiden Parteien ein verbindlicher Kaufvertrag abgeschlossen war.

Die im Vergleich zu anderen Medien höheren Herstellungskosten machten Fotografien zu wertvollen Abbildungen. Mindestens ebenso wichtig war, dass es sich bei ihnen um das modernste visuelle Medium seiner Zeit handelte, das selbst ein Symbol des Fortschritts war. ${ }^{103}$ Nach der offiziellen weltweiten Vorstellung des fotografischen Verfahrens 1839 dauerte es nur etwa 25 Jahre, bis die ersten Fabrikanten sich mit Aufträgen an Fotografen wandten. Damit

99 Beispielsweise: Akcionernoe obščestvo russkich ėlektrotechničeskich zavodov Simens i Gal'ske: Ėlektričeskie mostovye krany, in: Zapiski imperatorskago russkago techničeskago obščestva, 1899, Heft 1, S. 45 .

100 Genauer hierzu: Kapitel „Fabriken als Konsumartikel - Illustrierte Zeitschriften“, S. 241-282.

101 Pugh: Industrial Image, S. 17.

102 Beispielsweise CGA Moskvy f. 318, op. I t 1, d. 258, l. 18-19; f. 318, op. I t 1, d. 393, l. 45-46; f. 318, op. I t 1, d. 789, l. 72-75; f. 318, op. I t 1, d. 1051, l. 14-15; f. 318, op. I t 1, d. 1451, l. 93-94, 97 .

103 Assegond: La photographie du travail, S. 201; Starl: Im Prisma des Fortschritts, S. 10; Susanne Witzgall: Die unsichtbare Industrie. Tiroler Unternehmen in zeitgenössischer Fotografie, in: Günther Moschig; Gabriele Rath; dies. (Hrsg.): Industrielle Bildwelten. Tiroler Industrie in zeitgenössischer Fotografie, Innsbruck 2007, S. 9-21, S. 10. 
präsentierten die Abzüge die Unternehmer in doppelter Hinsicht als innovativ: einerseits über die Motive von Maschinen und modernen Produktionsanlagen, andererseits über das gewählte Medium der Darstellung, der Fotografie. Fabrikbesitzer nutzten diese Möglichkeit, um zu demonstrieren, wie offen sie neuen technischen Entwicklungen gegenüber stünden, woraus der Betrachter folgern konnte, dass auch in der Produktion die neuesten Maschinen zum Einsatz kamen.

Gleichzeitig stellte die Fotografie ein Distinktionsmerkmal dar, mit dem sich Industrielle von ihren Konkurrenten abgrenzten. Die Assoziation von Moderne und Fotografie beschränkte sich nicht auf Originalabzüge, sondern galt auch für Reproduktions-, Druck- oder Bearbeitungstechniken von Fotografien. Bereits 1885 veröffentlichte die Russisch-Amerikanische Gummifabrik in St. Petersburg einen Katalog, in dem statt Stichen Phototypien die Produkte für den Kunden wiedergaben. ${ }^{104}$ Diese Technik nutzte eine Druckplatte auf Gelatinebasis, mit der allerdings nur zwischen 1.000 und 2.00o Mal gedruckt werden konnte, bevor die Platte erneuert werden musste. In einer Zeit, als Reproduktionen von Fotografien noch relativ teuer waren, machten diese Illustrationen die Publikation zu einem kostbaren und gleichzeitig fortschrittlichen Objekt, und dies nicht nur im Zarenreich. Die deutsche Firma Krupp veröffentlichte ihren ersten mit Lichtdrucken illustrierten Katalog erst 1892. ${ }^{105}$

Eine weitere Technik, die Fabrikanten besonders in aufwendigen Reklamepublikationen und Katalogen nutzten, war die Bearbeitung von Fotografien mit dem Luftpinsel, aerograf genannt (heute Airbrush). Es handelte sich um einen Retuschierapparat, bei dem Druckluft die Farbe durch eine Düse gleichmäßig und fein zerstäubte, so dass fließende Farbübergänge entstanden. Um klare Kanten zu erhalten, arbeiteten die Retuscheure mit Abdeckungen und Schablonen aus Papier. ${ }^{106}$ Dieses Verfahren war in den USA bereits in den 1870er Jahren in Fotoateliers verbreitet, um großflächige Retuschen

104 RNB OE்: E் ((Al Tch 440)/(3-1)): G. fan Gil'ze fan der Palc: Illjustrirovannyj Katalog. Vysočajše utverždennago tovariščestva rossijsko-amerikanskoj rezinovoj manufaktury učreždennago v 1860 gody v S. Peterburge, Sankt-Peterburg 1885 .

105 Rudolf Herz: Gesammelte Fotografie und fotografierte Erinnerungen. Eine Geschichte des Fotoalbums an Beispielen aus dem Krupp-Archiv, in: Klaus Tenfelde (Hrsg.): Bilder von Krupp. Fotografie und Geschichte im Industriezeitalter, München 1994, S. 241-267, S. $257-258$.

106 Chas. L. Burdick: Finishing Photographs with the Aerograph for Process Engraving, in: Penrose's Pictorial Annual,Jg. 9/1903-1904, S. 106-108, S. 107; G. Merkator: Fotografičeskaja retuš. Sovremennaja chimičeskija, mechaničeskija i optičeskija posobija. Raskrašivanie fotografii, Perevel s nemeckago G. K. Bujakovič, Sankt-Peterburg 190o, S. 44-45. 
durchzuführen. ${ }^{107}$ Es galt als besonders gut geeignet für den Kontext der Industriefotografie. ${ }^{108}$ In Russland setzte sich die Technik erst um die Jahrhundertwende durch, zu diesem Zeitpunkt beschrieben auch Autoren in fotografischen Handbüchern das Verfahren. ${ }^{109}$ Die entsprechend bearbeiteten Bilder eigneten sich besonders gut, um aus ihnen Druckplatten für Autotypien herzustellen, was unter anderem ein Grund dafür war, dass diese Reproduktionsform beziehungsweise diese Form der Retusche ${ }^{110}$ in der Industrie eine besondere Popularität erlangte. In England, Deutschland sowie im Zarenreich ließen Unternehmer Fotografien ihrer Betriebe entsprechend bearbeiten, und fotografische Zeitschriften gaben Hilfestellungen, wie ein besonders gutes Ergebnis erzielt werden könne. ${ }^{111}$ Die englische Zeitschrift Penrose's Pictorial Annual veröffentlichte eine Illustration, deren eine Hälfte die Abbildung nach der Überarbeitung mit dem Luftpinsel, die andere Hälfte die originale Fotografie zeigt (Abb. 3).

Hier werden einige Vorteile der Technik deutlich: Die Arbeit des Retuscheurs ermöglichte es, den Industriellen die Objekte in eine fiktive, idealisierte Umgebung zu transferieren - so ersetzen rechts weiße Fließen den ursprünglichen Fußboden. Unerwünschte Bildelemente konnten getilgt und entsprechend die Charakteristika der Produkte und Maschinen betont werden. Mit dieser Bearbeitungstechnik ging einher, dass Fotografien nicht mehr als Abbild der Realität gelesen werden konnten. Dies war jedoch für die Verwendung in Firmenpublikationen nicht wesentlich. Vielmehr war in diesem Kontext entscheidend, dass es sich um eine neue Technik handelte, die überdies zeitaufwendig und teuer war. Damit entsprach die Technik einer Forderung zeitgenössischer Werbespezialisten, die betonten: Das Ziel der Reklame müsse sein, dass der Empfänger sie als Geschenk auffasse und aufbewahre. Scheue das Unternehmen die damit verbundenen Ausgaben für hochwertiges Papier und Druck, schade die Werbung dem Betrieb mehr, als dass sie nutze.112

107 G. H. Emmerich (Hrsg.): Lexikon für Photographie und Reproduktionstechnik (Chemigraphie, Lichtdruck, Heliogravüre), Wien, Leipzig 1910, S. 9.

108 Peter Stebbing: Industrial Image 1918 to 1986, in: Sue Davies; Caroline Collier (Hrsg.): Industrial Image. British Industrial Photography 1843 to 1986 , London 1986, S. $36-55$, S. 41; Christian Burchard: Fotografie oder Stich. Der Wettstreit der Drucktechniken in Firmenschriften zwischen 1890 und 1930, in: Irene Ziehe; Ulrich Hägele (Hrsg.): Gedruckte Fotografie. Abbildung, Objekt und mediales Format, Münster 2015, S. 250-265, S. 259-26o.

109 Merkator: Fotografičeskaja retuš', S. 44-49.

110 Emmerich (Hrsg.): Lexikon für Photographie, S. 6.

111 Burdick: Finishing Photographs, S. 106-108.

112 o. A.: Iskusstvo reklamirovat', S. 43, 51. 


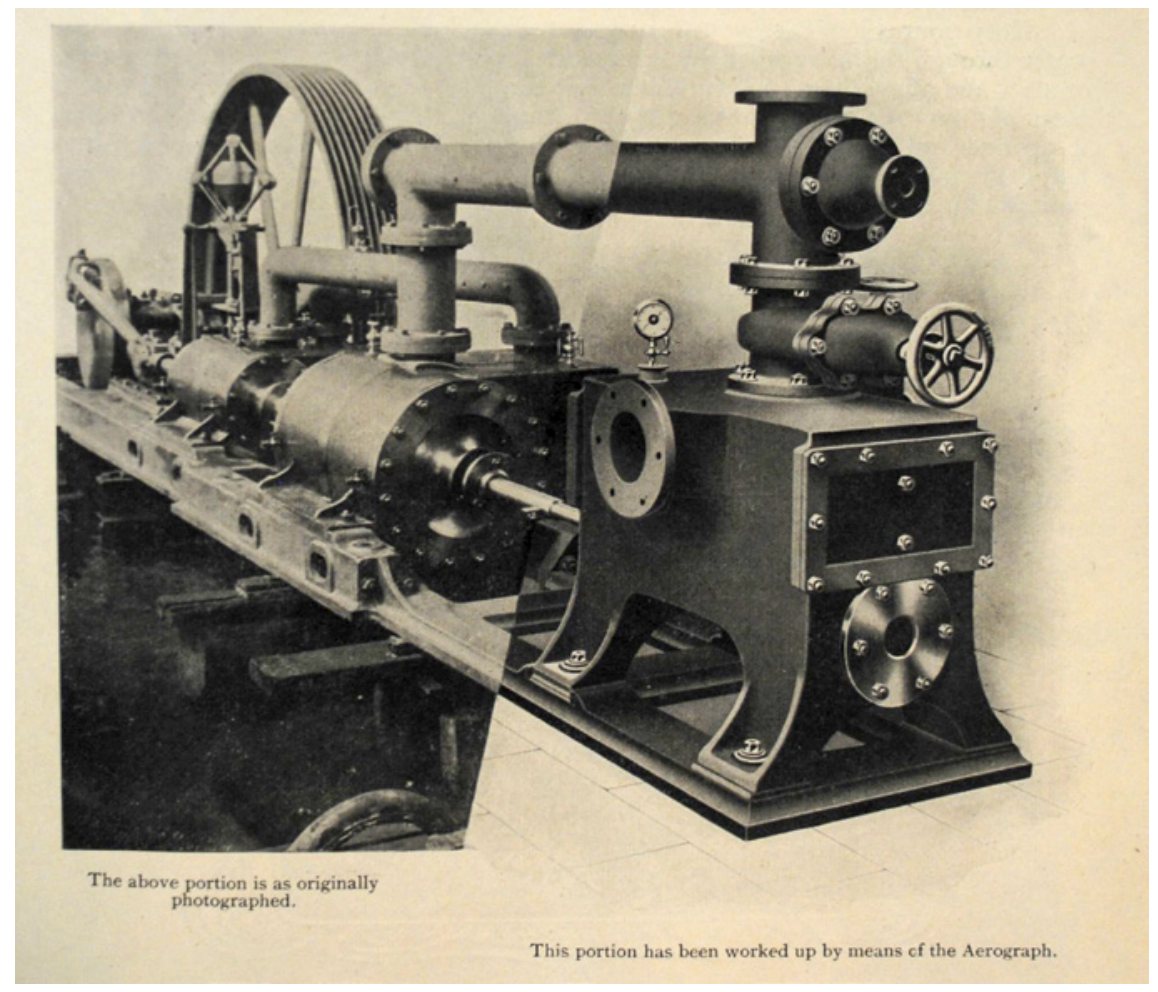

Abb. 3

o. A.: o. T., in: Chas. L. Burdick: Finishing Photographs with the Aerograph for Process Engraving, in: Penrose's Pictorial Annual, Jg. 9/1903-1904, S. 106-108, S. 107.

\subsection{Fazit}

Die ersten Annäherungsversuche zwischen Industrie und Fotografie fanden bereits wenige Jahre, nachdem Daguerre das Verfahren bekanntgegeben hatte, statt. Aus vereinzelten Kooperationen entwickelte sich eine bis heute anhaltende Partnerschaft, von der beide Parteien profitierten. Das neue Bildmedium veränderte Arbeitsweisen innerhalb der Fabriken nachhaltig und machte sich damit zum unentbehrlichen Begleiter. Auch für die internen Produktionsabläufe spielten fotografische Aufnahmen eine wichtige Rolle: in der Dokumentation, aber auch im Bereich der Ausbildung oder dem Umgang mit Katastrophen. Gleichzeitig waren Fotografien wertvolle Objekte, mit denen Unternehmen insbesondere ihre Selbstpräsentation in der russischen und internationalen Öffentlichkeit, beispielsweise auf Ausstellungen, illustrierten. In Verbindung mit den neu entstandenen Reklametechniken nutzten 
Fabrikanten die Abbildungen, um sich von der wachsenden Konkurrenz abzugrenzen und ihre eigene Aufgeschlossenheit gegenüber technischen Neuerungen zu demonstrieren. Außerdem konnten sie über die Wahl des Mediums der Fotografie sowie neuer Retuschetechniken unterstreichen, dass sie für ihre Kundschaft keine Kosten und Mühen scheuten. 